\title{
Dynamics of ground-state reverse proton transfer in the 7-azaindole/carboxylic acid systems
}

\author{
Wei-Ping $\mathrm{Hu}^{\text {a,*}, ~ R u-M i n ~ Y o u ~}{ }^{\text {a }}$, Shih-Yao Yen ${ }^{\text {a }}$, Fa-Tsai Hung ${ }^{\text {b }}$, \\ Po-Hung Chou ${ }^{\text {c, }}$ Pi-Tai Chou ${ }^{\text {c,1 }}$ \\ ${ }^{a}$ Department of Chemistry and Biochemistry, National Chung-Cheng University, Chia Yi 621, Taiwan, ROC \\ ${ }^{\mathrm{b}}$ National Hu-Wei Institute of Technology, Yunlin, Taiwan ROC \\ c Department of Chemistry, National Taiwan University, Taipei, Taiwan ROC
}

Received 24 September 2002; in final form 15 November 2002

\begin{abstract}
Dual-level direct dynamics calculation of the ground-state proton transfer reaction is reported for the 7-azaindole(7AI)/carboxylic acid system. The reaction path was calculated and the two-dimensional potential energy surface scan was performed at various levels of theory. Only one transition state geometry was resolved in the ground state from the ab initio calculation. The zero-point corrected barrier for the reverse proton transfer was found to be small $(\sim 0.2 \mathrm{kcal} / \mathrm{mol})$. The calculated reverse rate constant $\left(1.45 \times 10^{12} \mathrm{~s}^{-1}\right)$ at $300 \mathrm{~K}$ is significantly higher than the rate of tautomer emission $\left(3.8 \times 10^{8} \mathrm{~s}^{-1}\right)$ from the $\mathrm{S}_{1}^{\prime}$ excited state.
\end{abstract}

(c) 2003 Elsevier Science B.V. All rights reserved.

\section{Introduction}

The excited-state double proton transfer (ESDPT) reaction in the 7-azaindole (7AI) dimer has long been recognized as a prototype to mimic the photoinduced mutation of the DNA base pair [1]. The large separation (see Fig. 1) between N(1)-H proton and pyridinic nitrogen $(\mathrm{N}(7))$ in 7AI prohibits the intramolecular single-proton-transfer process. Accordingly, proton transfer takes place through the assistance of guest molecules pos-

\footnotetext{
${ }^{*}$ Corresponding author. Fax: +886-5-272-1040.

E-mail address: chewph@ccu.edu.tw (W.-P. Hu).

${ }^{1}$ Also corresponding author.
}

sessing bifunctional properties. For 7AI/guest hydrogen-bonded (HB) complexes, the energetics and structures of the proton-bridged relay as well as the reaction dynamics through the proton conduit have received considerable attention. Recently, femtosecond techniques have actively participated in studying the dynamics of ESDPT in the 7AI dimer [2-6]. Although the interpretation is somewhat scattered in terms of a one-step $[2-4,7,8]$ versus two-step $[5,6,9]$ mechanism, kinetic isotope effects on the rate of ESDPT are clearly resolved and a small energy barrier has been deduced. In another approach, in contrast to the prohibition of excited-state proton transfer for the isolated 1:1 and 1:2 7AI/ $\mathrm{H}_{2} \mathrm{O}$ complexes in the cold beam $[10,11]$, the remarkable excited-state proton- 

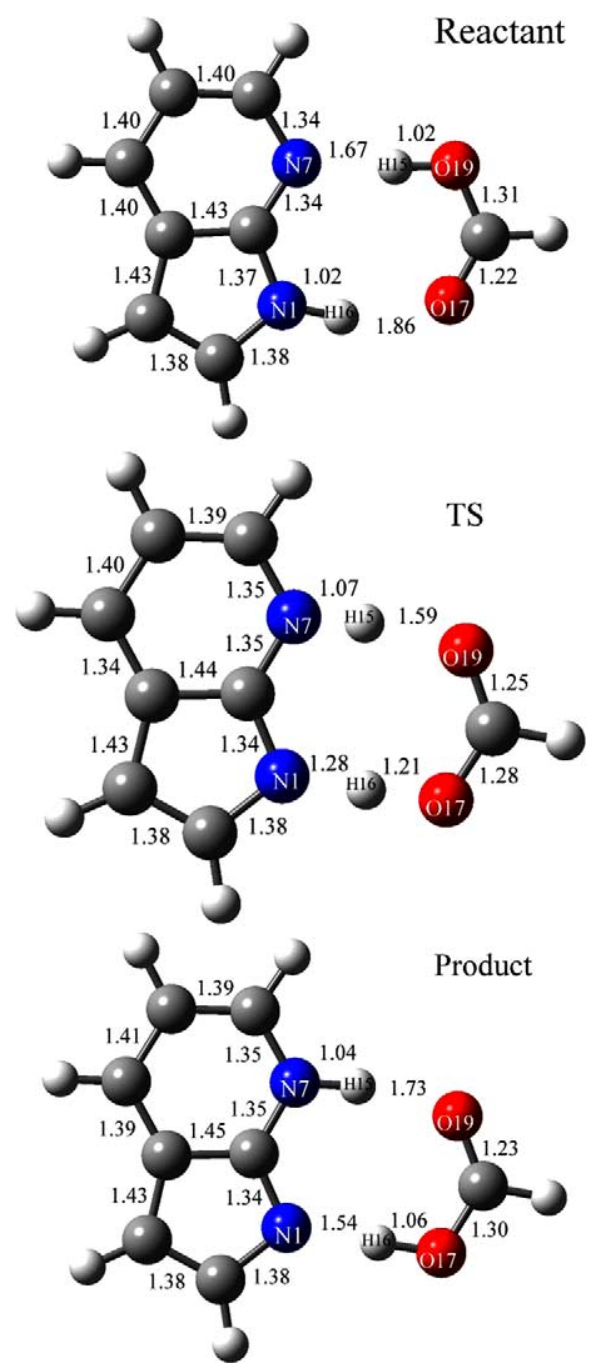

Fig. 1. The structures of the reactant complex, the product complex, and the double proton transfer transition state for the 7AI/formic acid system calculated at B3LYP/6-31G( $\left(\mathrm{d}^{\prime}, \mathrm{p}^{\prime}\right)$ level. The bond lengths are in angstroms.

transfer tautomerism for 7AI in bulk protic solvents $[1,12-15]$ implies that solvation stabilizations play a key role in proton transfer dynamics. In the gas phase, Chaban and Gordon [16] calculated that the presence of one water molecule significantly reduces the excited-state proton transfer barrier to $\leqslant 6 \mathrm{kcal} / \mathrm{mol}$. The addition of more than one water molecule should result in a further lowering of the activation energy. Siebrand and co-workers [17] shared the same viewpoint and suggested that model studies involving more solvent molecules are needed before reaching a basic understanding of the proton transfer process in bulk water. Further direct dynamic approaches to the water catalyzed proton transfer in 7AI have been reported with a modified instanton method [18], in which the multidimensional trajectory along the reaction potential energy surface (PES) has been systematically simplified. In another approach, a molecular dynamic simulation in bulk water performed by Mente and Maroncelli [19] led to a conclusion in favor of the slow ESDPT reaction dynamics ( $\sim$ a few hundreds ps) from the entire 7AI solvated species that exists predominantly as a 1:2 $\left(7 \mathrm{AI} / \mathrm{H}_{2} \mathrm{O}\right)$ 'neighbor-bonded' structure. This viewpoint was recently supported by analyzing both the rise and decay dynamics of the dual emission for a 7AI analogue, 3-cyano-7azaindole, in $\mathrm{H}_{2} \mathrm{O}$ [20].

An equally important approach is to gain detailed insights into the dynamics of ground-state proton transfer, which might provide the fundamental basis for the biological process where external HB bridges are necessary for the proton transfer reaction. Unfortunately, except for the 7AI dimer, to our knowledge, dynamic approaches of the ground-state reverse proton transfer (GSRPT) for 7AI/guest systems have not yet been reported. The lack of real time GSRPT dynamics makes theoretical approaches intriguing and significant. In this study, both experimental and theoretical attempts have been made to investigate the ground-state proton transfer dynamics on the 7AI/carboxylic acid HB system. The 7AI/carboxylic acid dual HB complex offers several advantages over the 7AI dimer [21] or other corresponding hydrogen-bonded complexes [16-18] with respect to theoretical approaches. In comparison to the 7AI dimeric system, the relatively small molecular framework in, e.g., the 7AI/formic or acetic acid complex allows a more accurate dynamic approach based on higher levels of theory. Conversely, unlike $7 \mathrm{AI} / \mathrm{H}_{2} \mathrm{O}$ or $7 \mathrm{AI} /$ alcohol complexes requiring the aforementioned second-shell solvent stabilization for the transition state (TS) or intermediate [16-18], the formation of a 1:1 7AI/acetic acid complex has been reported to undergo a fast rate of ESDPT in nonpolar solvents [22]. One thus 
expects similar ground-state proton transfer dynamics free from solvent perturbations, simplifying the theoretical approaches. Furthermore, the dissociation of the 7AI(T) dimer (hereafter $(\mathrm{T})$ denotes the proton-transfer tautomer) in the excited state has been reported, giving rise to the 7AI(T) monomer species that undergoes slow GSRPT dynamics (vide infra) [23]. This complication can be avoided in the $7 \mathrm{AI} /$ acetic acid system due to its large association constant [22].

\section{Experimental section}

\subsection{Theoretical methods}

Note that formic acid instead of acetic acid was applied as the guest molecule in the theoretical approach in order to reduce the computational demand. Such a replacement should make only small differences in the results due to the similarity in the two molecular structures, in particular the carboxylic functional group.

The structures of the reactant complex, the product complex, and the double proton-transfer transition state in the electronic ground state were calculated using the PM3 semiempirical method and the Hartree-Fock and B3LYP hybrid density functional theory with a $6-31 \mathrm{G}\left(\mathrm{d}^{\prime}, \mathrm{p}^{\prime}\right)$ basis set. We also calculated two dimensional potential energy maps by varying the $\mathrm{N}-\mathrm{H}$ and $\mathrm{O}-\mathrm{H}$ bond distances involved in the hydrogen transfer in a relaxed scan calculation at both the PM3 and HF/6-31G(d $\left(\mathrm{d}^{\prime}, \mathrm{p}^{\prime}\right)$ levels. Single point energy calculations were performed at the MP2/ $6-31+\mathrm{G}\left(\mathrm{d}^{\prime}, \mathrm{p}^{\prime}\right)$ and QCISD/6-31G( $\left(\mathrm{d}^{\prime}, \mathrm{p}^{\prime}\right)$ levels at the B3LYP/6-31G $\left(\mathrm{d}^{\prime}, \mathrm{p}^{\prime}\right)$ stationary point geometries.

In the dynamics modeling, we performed duallevel variational transition state theory (VTST) $[24,25]$ calculations at the canonical variational theory (CVT) [24] level. The minimum energy paths (MEP) [24] of the reaction and the deuterated reaction (only the two hydrogen atoms being transferred are deuterated) were calculated at B3LYP/6-31G $\left(\mathrm{d}^{\prime}, \mathrm{p}^{\prime}\right)$ levels of theory with gradient and hessian steps of 0.005 and 0.025 bohr, respectively. The scaling mass was set to $1 \mathrm{amu}$. The
MEP was calculated from -1.2 to 1.8 bohr. The MEP of the perprotic reaction was also calculated at the HF/6-31G $\left(\mathrm{d}^{\prime}, \mathrm{p}^{\prime}\right)$ level for comparison. The barrier width was calibrated using the SIL-2 scheme [26] based on the energy profile calculated at the MP2/6-31+ G(d $\left.\mathrm{d}^{\prime}, \mathrm{p}^{\prime}\right)$ level along the B3LYP/ 6-31G $\left(\mathrm{d}^{\prime}, \mathrm{p}^{\prime}\right)$ reaction path. The QCISD/6-31G $\left(\mathrm{d}^{\prime}, \mathrm{p}^{\prime}\right)$ single-point results were used for the highlevel energies in the dual-level VTST calculation. Tunneling effects were considered by applying the microcanonical optimized multidimensional tunneling ( $\mu \mathrm{OMT})$ approximation [27]. The electronic structure calculation was performed using the GAUSSIAN 98 program [28] and the VTST calculation was performed using the GaussRate 8.2 program [29].

\subsection{Measurements}

The nanosecond transient absorption was recorded with a laser flash photolysis system (Edinburgh LP920), in which the 4th harmonic (266 $\mathrm{nm})$ of an Nd:YAG laser (266 nm) and a whitelight square pulse were used as pump and probe beams, respectively. These two pulses were crossed at a $90^{\circ}$ angle with an overlapping distance of $\sim 10 \mathrm{~mm}$. The temporal resolution was limited by the excitation pulse duration of $\sim 6 \mathrm{~ns}$. The transient absorption system has been optimized by studying GSRPT of 7-hydxoyquinoline (7HQ) in methanol. Upon excitation 7HQ undergoes a solvent (e.g., methanol) catalyzed proton transfer, giving rise to a keto-tautomer species. The ground state keto-tautomer is long-lived ( $\tau \sim 4.2 \mu \mathrm{s}$ ) with a transient absorption maximized at $430 \mathrm{~nm}$ [30]. Using 7HQ as a standard reference, a transient absorbance as low as $\sim 1.0 \times 10^{-3}$ could be achieved in the current system with an average of 100 laser shots.

The setup of two-step laser induced fluorescence (TSLIF) measurements has been explained in our previous report [23]. In this study, a 4th harmonic of the Nd:YAG served as a pump pulse, while an Nd:YAG laser $(355 \mathrm{~nm})$ pumped optical parametric oscillator was used as the probe pulse, of which the wavelengths, after second harmonic generation, could be tuned in the range of 400-460 $\mathrm{nm}$. Occasionally, a (3rd harmonic $(355 \mathrm{~nm})$ of the 
YAG laser was used as a probe pulse. The sample solution was either aerated or degassed through three freeze-pump-thaw cycles.

\section{Results and discussion}

\subsection{Experimental approaches}

Several excited-state proton transfer molecules such as 7-hydroxyquinoline [30], $o$-hydroxybenzaldehyde [31] undergo fast ( picoseconds) excited-state proton transfer, while the GSRPT is relatively much slower ( $\sim$ microseconds). Thus, the main goal of the experiments in this study is to search any long-lived ground-state tautomer species generated from the excited-state proton transfer reaction. It is worth noting that in the case of the 7AI dimer, an unexpectedly slow rate of GSRPT ( microseconds) was once reported [32]. However, later experiments based on spectral differences in the tautomer emission between steady state and TSLIF measurements concluded that the long-lived transient species originates from the $7 \mathrm{AI}(\mathrm{T})$ monomer generated by the dissociation of the $7 \mathrm{AI}(\mathrm{T})$ dimer during its excited-state life span. Accordingly, the intrinsic GSRPT in the 7AI(T) HB dimer is also too fast to be resolved [23]. For the case of the 7AI/acetic acid HB complex, the rate constant of tautomer $\mathrm{S}_{1}^{\prime} \rightarrow \mathrm{S}_{0}^{\prime}$ transition was measured to be $3.8 \times 10^{8} \mathrm{~s}^{-1}\left(\tau_{\mathrm{f}}=2.63 \times 10^{-9} \mathrm{~s}\right.$ [22]). If the rate of $\mathrm{S}_{0}^{\prime} \rightarrow \mathrm{S}_{0}$ ground state reverse proton transfer (GSRPT) is $\gg 3.8 \times 10^{8} \mathrm{~s}^{-1}$, it would be conventionally irresolvable because the rate determining step is attributed to the $S_{1}^{\prime} \rightarrow S_{0}^{\prime}$ relaxation. Thus, the possibility of slow ( $>$ few nanoseconds) GSRPT was first examined by nanosecond transient absorption experiments. In this study 7AI $\left(\sim 2.8 \times 10^{-4} \quad \mathrm{M}\right)$ containing $1.0 \times 10^{-2} \mathrm{M}$ acetic acid in degassed cyclohexane was prepared. Knowing the association constant of $\sim 1.8 \times 10^{4} \mathrm{M}^{-1}$ [22], $\sim 98 \%$ of 7AI was calculated to be in the form of $7 \mathrm{AI} /$ acetic acid complex. The absorbance at the excitation wavelength of $266 \mathrm{~nm}$ was measured to be $\sim 1.2$.

Under the system limit of $10^{-3}$ in absorbance (see experimental section) we however were unable to resolve any transient spectra for the 7AI/ acetic acid system in both degassed and aerated cyclohexane. The lack of any transient absorption in the degassed solution also indicates either negligible population efficiency or fast relaxation time («system response of $6 \mathrm{~ns}$ ) for the $7 \mathrm{AI}(\mathrm{T})$ triplet state at $298 \mathrm{~K}$. The dynamics of GSRPT in the 7AI/acetic acid system was also investigated by TSLIF, in which the probe wavelength was tuned throughout $400-460 \mathrm{~nm}$ (see experimental section). Similarly, no TSLIF signal, i.e., the transient tautomer emission maximum at $\sim 490 \mathrm{~nm}$, was resolved at a pump-probe delay time of $>6$ ns. Note that in the TSLIF experiment the prompt tautomer fluorescence generated by the pump pulse has been subtracted. Summarizing the experimental results, both transient absorption and TSLIF measurements indicated that the rate constant of GSRPT is much greater than that of the $7 \mathrm{AI}(\mathrm{T}) /$ acetic acid emission of $3.8 \times 10^{8} \mathrm{~s}^{-1}$.

\subsection{Theoretical approaches}

Table 1 lists the calculated energies of reaction, $\Delta E$, and barrier heights, $\Delta V^{\neq}$, at various levels of theory. The forward double proton-transfer reaction, i.e., $7 \mathrm{AI}(\mathrm{N}) /$ formic acid $\rightarrow 7 \mathrm{AI}(\mathrm{T}) /$ formic acid, is endoergic in the electronic ground state.

Table 1

Calculated energies ${ }^{\mathrm{a}}$ of reaction and barrier heights (in kcal/ $\mathrm{mol}$ ) for the $7 \mathrm{AI} /$ formic acid system at various levels

\begin{tabular}{lll}
\hline & $\Delta V^{\neq}$ & Erxn \\
\hline PM3 & $24.1,29.3^{\mathrm{b}}$ & 11.5 \\
$\mathrm{HF} / 6-31 \mathrm{G}\left(\mathrm{d}^{\prime}, \mathrm{p}^{\prime}\right)^{\mathrm{c}}$ & $16.7(13.6)$ & $10.9(11.0)$ \\
$\mathrm{B} 3 \mathrm{LYP} / 6-31 \mathrm{G}\left(\mathrm{d}^{\prime}, \mathrm{p}^{\prime}\right) / /$ & $7.8(4.8)$ & $7.0(6.6)$ \\
MP2/6-31+G( $\left(\mathrm{d}^{\prime}, \mathrm{p}^{\prime}\right) / / \mathrm{B} 3 \mathrm{LYP} /$ & 9.3 & 8.0 \\
6-31G $\left(\mathrm{d}^{\prime}, \mathrm{p}^{\prime}\right)$ & & \\
QCISD/6-31G $\left(\mathrm{d}^{\prime}, \mathrm{p}^{\prime}\right) / / \mathrm{B} 3 \mathrm{LYP} /$ & 11.8 & 9.2 \\
6-31G $\left(\mathrm{d}^{\prime}, \mathrm{p}^{\prime}\right)$ & & \\
\hline
\end{tabular}

\footnotetext{
${ }^{\text {a }}$ Born-Oppenheimer energies; values in parentheses include zero-point energy correction calculated at the same level of theory.

${ }^{\mathrm{b}}$ These values are for the energies of the first and second transition state (TS), respectively. The energy of the complex between the two TS is $20.9 \mathrm{kcal} / \mathrm{mol}$.

${ }^{\mathrm{c}} 6-31 \mathrm{G}\left(\mathrm{d}^{\prime}, \mathrm{p}^{\prime}\right)$ is a modified version of the $6-31 \mathrm{G}(\mathrm{d}, \mathrm{p})$ basis set developed by Petersson and coworkers, defined as part of the Complete Basis Set methods. See G.A. Petersson, M.A. Al-Laham, J. Chem. Phys. 94 (1991) 6081.
} 
The reaction energy calculated at our highest-level calculation [QCISD/6-31G( $\left(\mathrm{d}^{\prime}, \mathrm{p}^{\prime}\right)$ ] was $9.2 \mathrm{kcal} /$ mol. Using the PM3 semiempirical method we were able to locate two distinct transition states with barrier heights of 24.1 and $29.3 \mathrm{kcal} / \mathrm{mol}$. However, Hartree-Fock and B3LYP calculations showed only one transition state with barriers of 16.7 and $7.8 \mathrm{kcal} / \mathrm{mol}$, respectively. The barriers of the reverse reaction were predicted to be very low $(0.8-2.6 \mathrm{kcal} / \mathrm{mol})$ by calculations that explicitly include correlation energy. When the vibrational zero-point energies calculated at the B3LYP/ 6-31G( $\left(\mathrm{d}^{\prime}, \mathrm{p}^{\prime}\right)$ level are included, the reverse barrier disappears at the B3LYP and MP2 levels and it becomes only $0.2 \mathrm{kcal} / \mathrm{mol}$ at the QCISD level.

The calculated structures of the reactant, TS, and product at B3LYP/6-31G $\left(\mathrm{d}^{\prime}, \mathrm{p}^{\prime}\right)$ level are shown in Fig. 1. All optimized geometries are in the planar configuration. As shown in Fig. 1, the N7-H15 distance changes drastically from $1.67 \AA$ in the reactant to $1.07 \AA$ in TS, indicating that at TS, the first hydrogen transfer from the acidic hydrogen (H15) of the formic acid is nearly com- plete, while the other hydrogen (H16) being transferred is only at the midway point. The hydrogen bond distances of $\mathrm{H} 15-\mathrm{O} 19$ and $\mathrm{N} 1-\mathrm{H} 16$ in the product are on average $0.13 \AA$ shorter than those in the reactant (i.e., H16-O17 and N7-H15). The result correlates with the calculated 7AI-formic acid association (Born-Oppenheimer) energies of $16 \mathrm{kcal} / \mathrm{mol}$ for the normal (7AI) form and 21 $\mathrm{kcal} / \mathrm{mol}$ for the tautomer $(7 \mathrm{AI}(\mathrm{T}))$ form at B3LYP/6-31G(d', $\left.\mathrm{p}^{\prime}\right)$ level.

Figs. 2 and 3 show the contour plots obtained by two-dimensional relaxed PES scans via PM3 and $\mathrm{HF} / 6-31 \mathrm{G}\left(\mathrm{d}^{\prime}, \mathrm{p}^{\prime}\right)$ methods, respectively. The reaction paths calculated by the HF/6-31G $\left(\mathrm{d}^{\prime}, \mathrm{p}^{\prime}\right)$ and B3LYP/6-31G(d', $\left.\mathrm{p}^{\prime}\right)$ methods were also mapped in Fig. 3. As shown in Fig. 2, at the PM3 level the first TS corresponds to the first proton transfer process from the formic acid to 7AI. The reaction path then passes through an intermediate $3 \mathrm{kcal} /$ mol lower than the first TS in energy before passing a sharp turn leading to the second TS. However, at the HF/6-31G( $\left(\mathrm{d}^{\prime}, \mathrm{p}^{\prime}\right)$ level, as shown in Fig. 3, the reaction path only has a rather flat

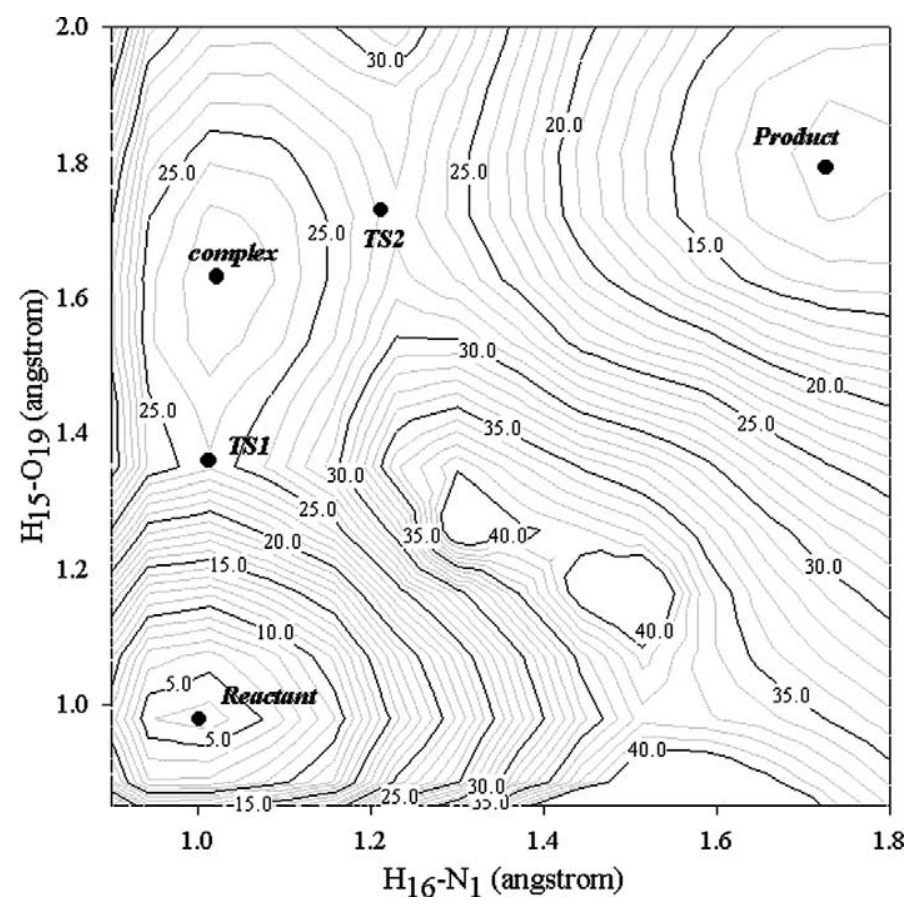

Fig. 2. Two dimensional potential energy map of the ground-state double proton transfer reaction in the 7AI/formic acid system calculated at PM3 level. The energies (in $\mathrm{kcal} / \mathrm{mol}$ ) are relative to that of the reactant. 


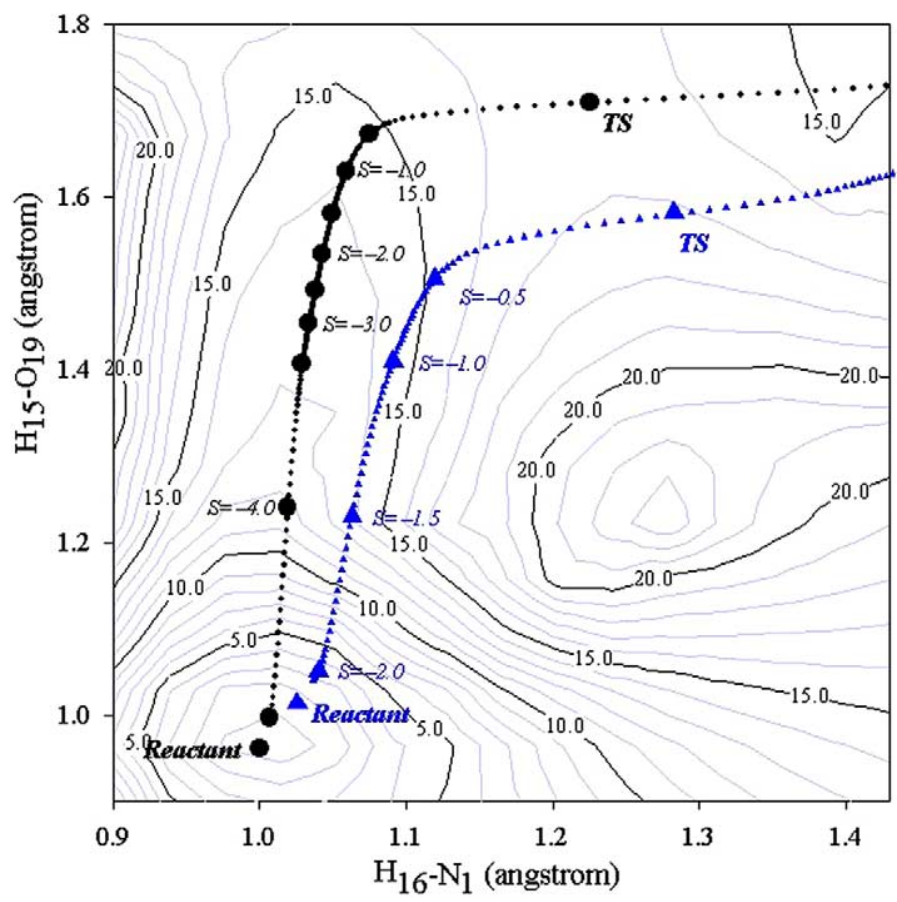

Fig. 3. Two dimensional potential energy maps at $\mathrm{HF} / 6-31 \mathrm{G}\left(\mathrm{d}^{\prime}, \mathrm{p}^{\prime}\right)$ level. The solid circles and triangles denotes the calculated reaction paths at HF6-31G(d $\left(\mathrm{d}^{\prime}, \mathrm{p}^{\prime}\right)$ and B3LYP/6-31G( $\left.\mathrm{d}^{\prime}, \mathrm{p}^{\prime}\right)$ levels, respectively. The energies (in $\mathrm{kcal} / \mathrm{mol}$ ) are relative to that of the reactant. The $s$ values are reaction coordinates in mass-scaled bohrs.

region, which corresponds to the first proton transfer process before the sharp turn leading to the only TS. The $\mathrm{B} 3 \mathrm{LYP} / 6-31 \mathrm{G}\left(\mathrm{d}^{\prime}, \mathrm{p}^{\prime}\right)$ reaction path lies on the lower-right side of the HF/ $6-31 \mathrm{G}\left(\mathrm{d}^{\prime}, \mathrm{p}^{\prime}\right)$ reaction path (see Fig. 3), indicating that the molecular geometry on the reaction path at the B3LYP level has longer $\mathrm{H} 16-\mathrm{N} 1$ and shorter H15-O19 bond distances.

The calculated ground-state forward reaction energy barrier $(8-12 \mathrm{kcal} / \mathrm{mol}$, depending on the levels of theory) of the current system is not high. Thus, the reaction should proceed readily at room temperature. However, due to its negligibly small energy barrier, the rate of reverse reaction is several orders of magnitude faster than that of the forward reaction. The calculated unimolecular forward and reverse rate constants on the ground electronic state and the corresponding deuterium kinetic isotope effects are listed in Table 2. As clearly shown in Table 2, the rate constant of the reverse reaction at, e.g., $300 \mathrm{~K}$ is larger than that of the forward reaction by more than six orders of magnitude. Variational effects [24] are strong in the current system, decreasing the TST rate constants by a factor of 2.4 at $300 \mathrm{~K}$. Conversely, the tunneling effects are found to be less important at room temperature, increasing the CVT rate constant by $55 \%$ at $300 \mathrm{~K}$. The relatively small tunneling effects are mainly due to the very low tunneling barrier since the energy of the TS is only slightly higher than that of the product (i.e., the 7AI(T)/formic acid complex). The small energy barrier also rationalizes the small temperature dependence in the reverse rate constants shown in Table 2. At the CVT/ $\mu$ OMT level, the deuterium kinetic isotope effects at $300 \mathrm{~K}$ were calculated to be 2.2 and 1.7 for the forward and reverse reactions, respectively. The reverse rate constant at 300 $\mathrm{K}$ was estimated to be $1.45 \times 10^{12} \mathrm{~s}^{-1}$ at the CVT/ $\mu \mathrm{OMT}$ level, which is $\sim$ three orders of magnitude larger than the lower limit of $3.8 \times 10^{8} \mathrm{~s}^{-1}$ [22] deduced from the decay rate of the $7 \mathrm{AI}(\mathrm{T}) /$ acetic acid emission following the excited-state proton transfer process. 
Table 2

Calculated proton-transfer rate constants for the $7 \mathrm{AI} /$ formic acid system in the ground state

\begin{tabular}{|c|c|c|c|c|}
\hline $\begin{array}{l}T \\
(\mathrm{~K})\end{array}$ & $\mathrm{TST}^{\mathrm{a}}$ & CVT & $\begin{array}{l}\text { CVT/ } \\
\mu \mathrm{OMT}\end{array}$ & $\mathrm{KIE}^{\mathrm{b}}$ \\
\hline \multicolumn{5}{|c|}{ Dual-level forward rate constants (in $\mathrm{s}^{-1}$ ) } \\
\hline 77 & $5.83(-14)^{\mathrm{c}}$ & $2.14(-15)$ & $6.52(-14)$ & 9.18 \\
\hline 100 & $4.33(-8)$ & $3.48(-9)$ & $3.99(-8)$ & 6.67 \\
\hline 200 & $3.04(2)$ & $8.82(1)$ & $2.13(2)$ & 3.20 \\
\hline 250 & $2.90(4)$ & $1.06(4)$ & $1.94(4)$ & 2.60 \\
\hline 300 & $6.11(5)$ & $2.56(5)$ & $3.97(5)$ & 2.22 \\
\hline 400 & $2.81(7)$ & $1.35(7)$ & $1.76(7)$ & 1.76 \\
\hline 600 & $1.34(9)$ & $7.08(8)$ & $7.98(8)$ & 1.33 \\
\hline 800 & $9.51(9)$ & $5.13(9)$ & $5.48(9)$ & 1.11 \\
\hline \multicolumn{5}{|c|}{ Dual-level reverse rate constants (in $\mathrm{s}^{-1}$ ) } \\
\hline 77 & $4.42(11)$ & $1.62(10)$ & 4.95 (11) & 3.37 \\
\hline 100 & $6.85(11)$ & $5.51(10)$ & $6.30(11)$ & 3.08 \\
\hline 200 & $1.56(12)$ & $4.54(11)$ & 1.09 (12) & 2.15 \\
\hline 250 & 1.92 (12) & 6.99 (11) & $1.28(12)$ & 1.87 \\
\hline 300 & $2.23(12)$ & $9.32(11)$ & $1.45(12)$ & 1.67 \\
\hline 400 & 2.78 (12) & $1.34(12)$ & 1.74 (12) & 1.40 \\
\hline 600 & 3.69 (12) & $1.95(12)$ & $2.20(12)$ & 1.12 \\
\hline 800 & $4.44(12)$ & 2.39 (12) & $2.56(12)$ & 0.98 \\
\hline
\end{tabular}

${ }^{\mathrm{a}}$ TST denotes conventional transition state theory.

${ }^{\mathrm{b}} \mathrm{KIE}=k_{\mathrm{H}} / k_{\mathrm{D}}$ where two hydrogens are substituted by deuterium.

${ }^{\mathrm{c}} 5.83(-14)$ means $5.83 \times 10^{-14}$.

\section{Conclusion}

In conclusion, we have made theoretical approaches to mimic the PES as well as dynamics of the ground-state double proton transfer in the 7AI/ formic acid system. At the ab initio Hartree-Fock level or upon incorporating electron correlation, we find only one reaction energy barrier, indicating a concerted, asynchronous double proton transfer reaction in which the reverse barrier is almost negligible. Temperature dependent multidimensional proton transfer rate constants were obtained using dual-level direct dynamics methods $[25,26]$. The rate constant of GSRPT was estimated to be on the order of $10^{11} \mathrm{~s}^{-1}$ at a temperature as low as $77 \mathrm{~K}$, which might experimentally be resolvable through the picosecond transient absorption incorporating stimulated emission-pumping technique [33]. It is however very difficult to achieve the 1:1 7HQ/guest dual HB complex formation in this type of cryogenic condition without interferences from aggregation [34]. Alternatively, an integrated system intrinsically composed of 7AI and a carboxylic group is proposed so that the intramolecular dual hydrogen bonds should serve as a prototype for the experimental approach. Focus on this is currently in progress.

\section{Acknowledgements}

Financial support from the National Science Council of ROC is gratefully acknowledged.

\section{References}

[1] C.A. Taylor, M.A. El-Bayoumi, M. Kasha, Proc. Natl. Acad. Sci. USA 63 (1969) 253.

[2] S. Takeuchi, T. Tahara, Chem. Phys. Lett. 277 (1997) 340.

[3] S. Takeuchi, T. Tahara, J. Phys. Chem. A 102 (1998) 7740.

[4] S. Takeuchi, T. Tahara, Chem. Phys. Lett. 347 (2001) 108.

[5] M. Chachisvilis, T. Fiebig, A. Douhal, A.H. Zewail, J. Phys. Chem. A 102 (1998) 669.

[6] T. Fiebig, M. Chachisvilis, M. Manger, A.H. Zewail, A. Douhal, I. Garcia-Ochoa, A. de La Hoz Ayuso, J. Phys. Chem. A 103 (1999) 7419.

[7] J. Catalán, P. Pérez, J.C. del Valle, J.L.G. de Paz, M. Kasha, Proc. Natl. Acad. Sci. USA 99 (2002) 5799.

[8] J. Catalán, J. Phys. Chem. A 106 (2002) 6738.

[9] D.E. Folmer, E.S. Wisniewski, S.M. Hurley, A.W. Castleman Jr., Proc. Natl. Acad. Sci. USA 96 (1999) 12980.

[10] Y. Huang, S. Arnold, M. Sulkes, J. Phys. Chem. 100 (1996) 4734.

[11] A. Nakajima, M. Hirano, R. Hasumi, K. Kaja, H. Watanabe, C.C. Carter, J.M. Williamson, T.A. Miller, J. Phys. Chem. A 101 (1997) 392.

[12] D. McMorrow, T. Aartsma, Chem. Phys. Lett. 125 (1986) 581.

[13] J. Koijnenberg, A.H. Huizer, C.A.O. Varma, J. Chem. Soc., Faraday Trans. 284 (1988) 1163.

[14] R.S. Moog, M. Maroncelli, J. Phys. Chem. 95 (1991) 10359.

[15] A.V. Smirnov, D.S. English, R.L. Rich, J. Lane, L. Teyton, A.W. Schwabacher, S. Luo, R.W. Thornburg, J.W. Petrich, J. Phys. Chem. B 101 (1997) 2758, and references therein.

[16] G.M. Chaban, M.S. Gordon, J. Phys. Chem. A 103 (1999) 185.

[17] Z. Smedarchina, W. Siebrand, A. Fernández-Ramos, L. Gorb, J. Leszczynski, J. Chem. Phys. 112 (2000) 566.

[18] A. Fernández-Ramos, Z. Smedarchina, W. Siebrand, M.Z. Zgierski, J. Chem. Phys. 114 (2001) 7518.

[19] S. Mentus, M. Maroncelli, J. Phys. Chem. A 102 (1998) 3860.

[20] P.T. Chou, W.S. Yu, C.Y. Wei, Y.M. Cheng, C.Y. Yang, J. Am. Chem. Soc. 123 (2001) 3599. 
[21] A. Douhal, V. Guallar, M. Moreno, J.M. Lluch, Chem. Phys. Lett. 256 (1996) 370.

[22] C.P. Chang, W.C. Hwang, M.S. Kuo, P.T. Chou, J.H. Clements, J. Phys. Chem. 98 (1994) 8801.

[23] P.T. Chou, W.S. Yu, Y.C. Chen, C.Y. Wei, S.S. Martinez, J. Am. Chem. Soc. 120 (1998) 12927.

[24] D.G. Truhlar, B.C. Garrett, Acc. Chem. Res. 13 (1980) 440.

[25] W.-P. Hu, Y.-P. Liu, D.G. Truhlar, J. Chem. Soc., Faraday Trans. 90 (1994) 1715.

[26] C.-H. Huang, R.-M. You, P.-Y. Lian, W.-P. Hu, J. Phys. Chem. A 104 (2000) 7200

[27] Y.-P. Liu, D.-H. Lu, A. Gonzalez-Lafont, D.G. Truhlar, B.C. Garrett, J. Am. Chem. Soc. 115 (1993) 7806.
[28] M.J. Frisch et al., Gaussian 98, Revision A.7, Gaussian Inc., Pittsburgh, PA, 1998.

[29] J.C. Corchado, Y.-Y. Chuang, E.L. Coitiño, D.G. Truhlar, Gaussrate-version 8.2, University of Minnesota, 1999.

[30] P.T. Chou, S.S. Martinez, Chem. Phys. Lett. 235 (1995) 463, and references therein.

[31] J. Konijnenberg, A.H. Huiser, C.A.G.O. Varma, J. Chem. Soc. Faraday, Trans 284 (4) (1988) 363.

[32] K. Tokumura, Y. Watanabe, M. Udagawa, M. Itoh, J. Am. Chem. Soc. 109 (1987) 1346.

[33] For example, see: T. Dzugan, J. Schmidt, T.J. Aartsma, Chem. Phys. Lett. 127 (1986) 336.

[34] H. Bulska, A. Chodkowska, J. Am. Chem. Soc. 102 (1980) 3259. 